\title{
From pluripotent stem cell to adipocyte
}

\author{
Qi-qun Tang ${ }^{1,2}, \mathrm{Xi} \mathrm{Li}^{1}$, Hai-yan Huang ${ }^{1}$ \\ ${ }^{1}$ Key Laboratory of Molecular Medicine, Ministry of Education, Fudan University, Shanghai 200032, China; \\ ${ }^{2}$ Institute of Biomedical Sciences, Fudan University, Shanghai 200032, China
}

Obesity leads to both an increase in adipocyte number (hyperplasia) and size (hypertrophy). The adipocyte developmental pathway can be resolved and studied in three distinct steps: 1) adipocyte lineage commitment; 2) mitotic clonal expansion; and 3) terminal differentiation into adipocytes. Hyperplasia is due to step 1\&2 while hypertrophy is due to step 3. All three steps can be mimicked and studied separately by using established stem and preadipocyte cell lines in culture.

C3H10T1/2 is a pluripotent stem cell line, it has the potential to develop bone, muscle and fat cells. We recently identified a factor and developed conditions that cause 10T1/2 stem cells to commit to the adipocyte lineage in cell culture. Moreover, this process can be recapitulated in vivo. Treatment of 10T1/2 stem cells with our usual adipocyte differentiation protocol does not provoke commitment or differentiation, as indicated by failure to express cytoplasmic triglyceride or adipocyte markers. Only after initial exposure to BMP4, followed by treatment with differentiation inducers, do C3H10T1/2 stem cells enter the adipose development pathway and give rise to cells that express the adipocyte phenotype. We have been able to verify these findings in an in vivo context.

3T3-L1 is a committed preadipocyte cell line.Treatment with a mixture of differentiation inducers, initiates a cascade of events including the synchronous reentry of the cell cycle for 2 rounds of mitosis, a process referred to as mitotic clonal expansion which is required for subsequent terminal adipocyte differentiation. Upon induction of differentiation, C/EBP $\beta$ is expressed immediately but is unable to bind DNA and thus cannot yet function as a transcriptional activator. Acquisition of DNA binding activity is delayed until the cells traverse the G1-S checkpoint of the mitotic clonal expansion, at which point DNA binding activity is acquired. Following mitotic clonal expansion, C/EBP $\beta$ initiates a cascade of transcriptional activation. C/EBP $\beta$ activates expression of the C/EBP $\alpha$ and PPAR genes, which function together as pleiotropic transcriptional activators of the large group of genes that produce the adipocyte phenotype. We recently found that $\mathrm{C} / \mathrm{EBP} \beta$ is sequentially phosphorylated before activated, both ex vivo and in vitro experiments with $\mathrm{C} / \mathrm{EBP} \beta$ show that it is immediately undergoes a phosphorylation on Thr188 by MAPK/ERK and then by cdk2/CyclinA, phosphorylation of Thr-188 "primes" C/EBP $\beta$ for subsequent phosphorylation on Ser-184 and Thr-179 by glycogen synthase kinase 3b. Fully phosphorylation of C/EBP $\beta$ leads to the acquisition of DNA-binding function, and transactivation of the C/EBP $\alpha$ and PPAR $\gamma$ genes, thereby progression of terminal differentiation.

Keywords: phosphorylation, C/EBP $\beta, \mathrm{BMP} 4$

Cell Research (2008) 18:s96. doi: 10.1038/cr.2008.186; published online 4 August 2008

Correspondence: Qi-qun Tang

E-mail: qqtang@shmu.edu.cn

Dr Qi-Qun Tang received his Bachelor degree of Medicine in 1990 and $\mathrm{PhD}$ degree in Biochemistry and Molecular Biology in 1995 from Shanghai Medical University. He joined Dr M Daniel Lane's lab as a postoctoral fellow at the Department of Biological Chemistry at Johns Hopkins University School of Medicine in late 1995, and was promoted to faculty rank as a faculty of Research Associate in 1997. In 2002 he was appointed as an Assistant Professor in the Department of Pediatrics (Division of Endocrinology) with a joint appointment as an Assistant Professor in the Department of Biological Chemistry at Johns Hopkins University School of Medicine. He is now a Professor and Director for the Key Laboratory of Molecular Medicine (Ministry of Education) and Department of Biochemistry and Molecular Biology at Fudan University Shanghai Medical School. He also serves as the Vice-President for the Institutes of Biomedical Sciences and the Associate Dean for the Shanghai Medical School of Fudan University. 